\title{
Tree Versus Geometric Representation of Tests and Items
}

\author{
Michal Beller \\ National Institute for Testing and Evaluation, Israel
}

Factor-analytic techniques and multidimensional scaling models are the traditional ways of representing the interrelations among tests and items. Both can be classified as geometric approaches. This study attempted to broaden the scope of models traditionally used, and to apply an additive tree model (ADDTREE) that belongs to the family of network models. Correlation matrices were obtained from three studies and were analyzed using two representation models: Smallest Space Analysis (SSA), which is a multidimensional scaling model, and ADDTREE. The results of both analyses were compared for the two criteria of goodness of fit and interpretability. To enable a comparison with the more traditional factor-analytic approach, the data were also subjected to principal components analyses. ADDTREE fared better in both comparisons. Moreover, ADDTREE lends itself readily to an interpretation in terms of hierarchical cluster structure, whereas it is difficult to interpret SSA's dimensions. ADDTREE's close fit to the data and its coherence of presentation make it a convenient means of representing tests and items. Index terms: additive trees, ADDTREE, factor analysis, hierarchical clustering, multidimensional scaling, Smallest Space Analysis.

Much theoretical and empirical work in psychology uses proximity data, from which the structure in the interrelationships among a set of objects is derived. Several approaches and techniques have been developed to achieve this goal. The most

APPLIED PSYCHOLOGICAL MEASUREMENT

Vol. 14, No. 1, March 1990, pp. 13-28

(C) Copyright 1990 Applied Psychological Measurement Inc. 0146-6216/90/010013-16\$2.05 prevalent is the geometric approach, in which objects are represented as points in a continuous multidimensional space, such that the order of the proximities between the objects is reflected by the order of the proximities between the corresponding points (e.g., Coombs, 1964; Guttman, 1965, 1968; Shepard, 1962a, 1962b, 1974, 1980). An alternative is the classificatory approach, in which objects are represented by discrete clusters (e.g., Carroll, 1976; Johnson, 1967; Sattath \& Tversky, 1977; Shepard \& Arabie, 1979). The clustering structure among a set of objects can be interpreted as representing their common and distinctive features, such that objects that have common features are clustered together, while different clusters reflect distinctive features (Sattath \& Tversky, 1977; Tversky, 1977).

There is a mutual correspondence between features and clusters. The relation between a feature and the corresponding cluster is essentially that between the meaning of a concept and the set of objects to which it applies. The clusters or features used to classify objects can be specified in advance or else derived from some measure of similarity or dissimilarity between the objects, using a suitable model. Conversely, a clustering model can be used to predict the observed dissimilarities between the objects (Sattath \& Tversky, 1987). Throughout this article the terms "clusters" and "common features" will be used interchangeably.

Several studies have attempted to compare the two approaches, using simulated or real datasets 
from different fields (e.g., Carroll, 1976; Carroll \& Pruzansky, 1980; Gati, 1979, 1982; Johnson \& Tversky, 1984; Pruzansky, Tversky, \& Carroll, 1982; Sattath \& Tversky, 1977; Tversky \& Hutchinson, 1986). There are several criteria for comparing different representation models, and for assessing which is more appropriate for a given dataset. The two major criteria, technical and theoretical respectively, are:

1. Goodness of fit: The degree of correspondence between the solution and the data.

2. The interpretability and theoretical interest of the emerging solution.

Although some proximity data may be adequately represented by both the geometric and the classificatory approaches, there are datasets for which one approach is more favorable than the other. The geometric approach seems to be more appropriate for perceptual stimuli (e.g., color, sound) that can be characterized by a few continuous dimensions. On the other hand, the classificatory approach seems to be more appropriate for conceptual stimuli characterized by a large number of discrete features, for example, semantic categories such as countries or fruits (Pruzansky et al., 1982; Tversky \& Hutchinson, 1986).

Research in the area of psychological testing has traditionally made extensive use of representation models (e.g., Cattell, 1971; Davison, 1985; Guilford, 1967; Horn, 1968; Schlesinger \& Guttman, 1969; Spearman, 1927; Thurstone, 1938; Tryon \& Bailey, 1970; Vernon, 1961). Such representations are commonly based on observed correlations among tests. Because correlation matrices are very dense and difficult to interpret, several methods have been suggested to represent them in simpler and more parsimonious ways. These methods have been used to enhance the understanding of the structure underlying test performance and to corroborate theories on the structure of intelligence (e.g., Guilford, 1967; Spearman, 1927; Thurstone, 1938; Vernon, 1961).

Different representation models may highlight different aspects of the data and can lead to different interpretations and theories (e.g., planes suggest continuous dimensions whereas trees suggest discrete clusters). In the area of aptitude and achievement testing, the geometric approach has been used almost exclusively, either in the form of factor analysis (e.g., Guilford, 1967; Harman, 1976) or by use of multidimensional scaling (MDS) techniques (e.g., Guttman, 1968; Schlesinger \& Guttman, 1969). Despite their differences, factoranalytic techniques and MDS models can both be classified as geometric models because they both embed the objects (tests) in some coordinate space, so that the distances between the points represent the similarity between the respective objects (or, in the present case, the correlation between tests). A comparison between MDS and factor analysis is discussed by several researchers (e.g., Davison, 1983, 1985; Shepard, 1972).

The major goal of the present study was to demonstrate the application of the classificatory approach to psychometric data and to compare this approach with the more traditional geometric models. More specifically, the additive-tree method (ADDTREE; Sattath \& Tversky, 1977) was used to represent the relationships among aptitude and achievement tests, and among sets of individual items extracted from such tests. ADDTREE analysis has been applied in some areas of cognitive psychology (e.g., Gati, 1979; Pruzansky et al., 1982), but this is the first attempt to use it to describe the intercorrelations among psychological tests. The tree representation, which has several attractive formal properties (e.g., each pair of objects is joined by a unique path), permits a parsimonious description and a convenient graphical display.

ADDTREE is a hierarchical clustering model that represents a distance matrix as an additive or "path length" tree-a generalization of the ultrametric tree (e.g., Johnson, 1967). A tree is a connected graph without cycles, in which the terminal nodes represent the objects. ADDTREE first constructs a tree structure based on a partition of all the quadruples of variables into pairs of immediate neighbors, and then computes least-squares estimates of the lengths of the links (Sattath \& Tversky, 1977). The distance between the objects is given by the length of the shortest horizontal path that joins them along the tree branches (the vertical line is included only for graphical convenience).

To enhance the interpretation of the structure, 
ADDTREE selects a root for the tree on the basis of the data. However, the distances in an additive tree do not depend on the choice of the root. A rooted tree representation can be viewed as a hierarchy of clusters, and it can be interpreted in terms of common and distinctive features (Tversky, 1977). Under such an interpretation, the length of each arc of the tree corresponds to the measure of the common features shared by the cluster of all the objects originating from that arc-and by them alone (i.e., common features not shared by objects in any of the other clusters). The terminal arcs of the tree correspond to the measure of unique features of the respective objects (Tversky, 1977; Tversky \& Sattath, 1979). The longer an arc, the more distinct the cluster originating from it.

The (horizontal) path-length distance between any two objects is the measure of their distinctive features (the sum of their unique features). Therefore, an object connected to the root of the tree by a relatively short arc is "central" in the sense that it is the "nearest neighbor" of the maximal number of objects (Tversky \& Hutchinson, 1986). This object can be viewed as one with relatively few distinctive features, hence it is relatively similar to all other objects. Note that features common to all objects are not represented in the tree. Thus the common features that appear in ADDTREE correspond to features shared by some, but not all, objects. Analysis of the interpretation of common and distinctive features in a tree is presented in Sattath and Tversky (1987).

It is instructive to focus on ADDTREE representation of the following two structures: (1) single factor-represented by a degenerated single-cluster tree, in which all objects are directly connected to the root (star), and (2) simplex correlation matrices-represented by a single-branch tree, along which all objects lie (chain). These two extreme cases represent the two different meanings of the term "unidimensionality". More regular structures would be represented by "richer" trees with several branches, clusters, and subclusters.

In contrast to ADDTREE, Smallest Space Analysis (SSA-I; Guttman, 1968) is a nonmetric MDs technique. This technique yields very similar results to those obtained by an alternative program - KYST
(Kruskal, Young, \& Seery, 1977). SSA was applied by Schlesinger and Guttman (1969) to represent the structure among aptitude and achievement tests. Their analysis yielded a two-dimensional space in which the tests were represented as points. This structure was labeled "radex" by the researchers, and was postulated to be a general representation of the relationships between aptitude and achievement tests.

The comparison between SSA and ADDTREE is of particular interest when applied to the psychometric area, because tree and geometric models have been compared systematically in other areas of cognitive psychology (e.g., Carroll \& Pruzansky, 1980; Tversky \& Hutchinson, 1986) and there are simulation data that provide a good basis for comparing them (e.g., Pruzansky et al., 1982). Because fit improves by adding parameters, it is important to note that the two models, ADDTREE and the two-dimensional geometric configuration (SSA/2D), have approximately the same number of parameters. In ADDTREE the distances between the objects are determined by at most $2 n-2$ parameters (arcs of the tree), where $n$ is the number of elements of the set (for a further discussion of this topic see Sattath \& Tversky, 1977; Tversky \& Sattath, 1979), whereas SSA/2D and SSA/3D have $2 n$ and $3 n$ parameters, respectively (Pruzansky et al., 1982). Furthermore, Pruzansky et al. (1982) have demonstrated that there is no inherent a priori advantage, in terms of goodness of fit, of one model over the other. They found that the "appropriate" model (i.e., using MDS to represent data generated from a plane and ADDTREE to represent data generated from a tree) was always superior to the "inappropriate" model. The MDS model fit plane data about as well as ADDTREE fit tree data, over a wide range of set sizes and error levels.

In this study, the representations derived from the ADDTREE analyses were compared with those obtained by SSA-I (Guttman, 1968; Lingoes, 1970). The comparisons were carried out with respect to the two criteria mentioned above (i.e., goodness of fit and interpretation) for several datasets from various tests and items. To enable comparisons between the results obtained from these two models and the more traditional factor-analytic approach, 
a principal components analysis was performed as well.

\section{Method}

\section{Datasets}

Matrices of correlations between tests and between test items were analyzed by the three methods. The data matrices were obtained from three sources. The first two datasets were gathered at the Hebrew University's testing unit. In order to broaden the scope of the comparison, an additional dataset was adopted from Hoger (1964). This is the dataset that was previously subjected to SSA-I, yielding the radex structure (Schlesinger \& Guttman, 1969).
Dataset A: The 1980 Psychometric Entrance Test to the Hebrew University (PET). The PET is a general scholastic aptitude test used for selecting students for the various faculties of the Hebrew University. The test comprises 230 items grouped into 21 homogeneous subtests according to content. Table 1 lists the name and the number of items for each subtest. A detailed description of the test and its validities can be found in Beller and Ben-Shakhar (1981).

The raw scores of 2,644 examinees constituted the basis for computing the correlations among subtests and among test items. Items were scored dichotomously and tests were scored as the sum of the items answered correctly with no correction for guessing.

Table 1

The PET Subtests (Dataset A)

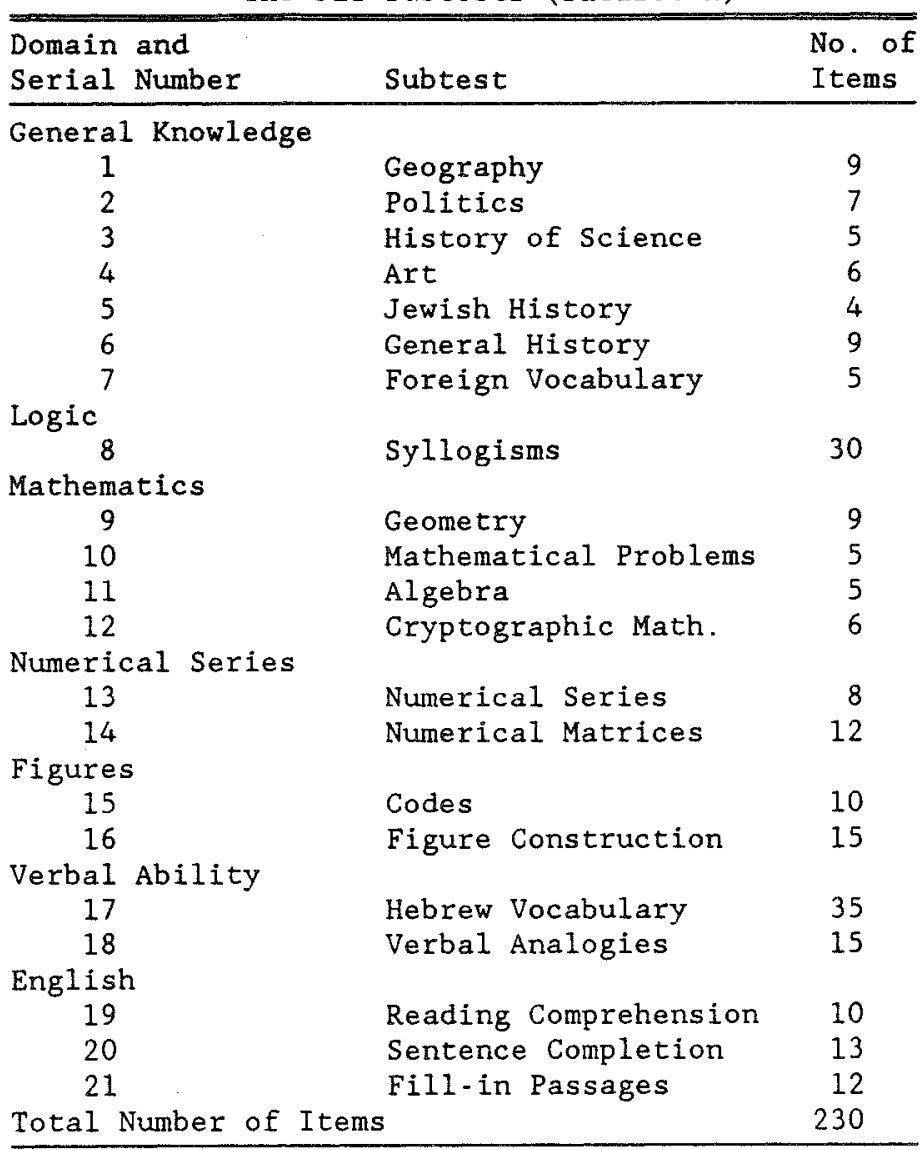


Table 2

The 14 Sets of Items Selected from the PET Test (Median Difficulty Levels: Easy $=.75$, Intermediate $=.47$, Difficult $=.34$ )

\begin{tabular}{|c|c|c|}
\hline Set & Subgroup & No. of Items \\
\hline \multicolumn{3}{|c|}{ Relatively Heterogeneous Items } \\
\hline a & Items selected from all 21 subtests & 62 \\
\hline $\mathrm{b}$ & 6 easiest items from each domain & 42 \\
\hline c & $\begin{array}{l}6 \text { items of intermediate difficulty from } \\
\text { each domain }\end{array}$ & 42 \\
\hline $\begin{array}{l}\mathrm{d} \\
\mathrm{e}\end{array}$ & $\begin{array}{l}6 \text { most difficult items from each domain } \\
15 \text { items each from: General Knowledge, }\end{array}$ & 42 \\
\hline $\mathbf{f}$ & $\begin{array}{l}\text { Hebrew Vocabulary, English } \\
15 \text { items each from: Logic, Mathematics, }\end{array}$ & 45 \\
\hline & Numerical Series & 60 \\
\hline \multicolumn{3}{|c|}{ Relatively Homogeneous Items } \\
\hline $\mathrm{g}$ & General Knowledge, all items & 45 \\
\hline h & Logic, all items & 30 \\
\hline i & Mathematics, a11 items & 25 \\
\hline j & Numerical Series, all items & 20 \\
\hline $\mathrm{k}$ & Figures, all items & 25 \\
\hline 1 & Hebrew Vocabulary, all items & 35 \\
\hline $\mathbf{m}$ & Verbal Analogies, all items & 15 \\
\hline $\mathrm{n}$ & English, all items & 35 \\
\hline
\end{tabular}

For the purpose of exploring the relationships among individual items, 14 different sets of items were selected from the 230 items. These sets of items are described in Table 2. Yule's $Q$ measure (also known as Goodman-Kruskal gamma) of predictive association for categorical data (Hayes, 1981) was computed between each pair of items within each set. This measure was chosen because it is relatively insensitive to marginal distributions (i.e., the difficulty levels of the items).

Dataset B: Validation study. These data were taken from a follow-up study of students, conducted at the Faculty of Social Sciences at the Hebrew University (Beller \& Ben-Shakhar, 1981), in which the predictive validities of 11 tests were investigated using the academic achievement score (GPA) at the end of the freshman year (1975) as the criterion. The 11 predictors included 7 general aptitude tests taken from the PET (1974) and 4 achievement tests, taken from the Israeli high school matriculation tests which, together with the criterion, are listed in Table 3.

Dataset C: Hoger study. Hoger (1964) gath-
Table 3

Variables Included in the Validation Study (Dataset B)

\begin{tabular}{|c|c|}
\hline $\begin{array}{l}\text { Domain and } \\
\text { Serial Number }\end{array}$ & Variable \\
\hline \multicolumn{2}{|c|}{ Matriculations (Achievement Tests) } \\
\hline 1 & English \\
\hline 2 & Hebrew \\
\hline 3 & Bible \\
\hline 4 & Mathematics \\
\hline \multicolumn{2}{|c|}{ Aptitude Tests (PET) } \\
\hline 5 & Figures \\
\hline 6 & Logic \\
\hline 7 & Memory \\
\hline 8 & General Knowledge \\
\hline 9 & Hebrew Vocabulary \\
\hline 10 & English \\
\hline 11 & Mathematics \\
\hline \multicolumn{2}{|c|}{ Freshman Year Grades } \\
\hline 12 & Criterion: GPA \\
\hline
\end{tabular}

ered information about the performance of 519 high school students in Germany, using 20 subtests (9 general aptitude tests and 11 achievement tests), 
as listed in Table 4. This dataset was chosen because it had been previously analyzed using SSA-I (Schlesinger \& Guttman, 1969).

\section{Analysis}

Each dataset was analyzed using two different scaling programs: SSA-I (Lingoes, 1970) in both two and three dimensions, and ADDTREE/P (Corter, 1982). For each scaling solution, two goodnessof-fit measures, $R_{L}^{2}$ and $R_{M}^{2}$, were used (Kruskal, 1964). $R_{L}^{2}$ is the squared product-moment correlation between the solution (the distances between the variables in the model) and the data (the correlations between the subtests), that is, the proportion of linear variance accounted for by the solution. $R_{M}^{2}$ is the proportion of variance explained by the best monotone correlation between the solution and the data. These measures were chosen because they are not altered by adding a constant to all the distances. Note that ADDTREE often adds

Table 4

The Subtests of Hoger's Study (Dataset C)

\begin{tabular}{cl}
\hline Domain and & \\
Serial Number & Subtest \\
\hline Aptitude Tests & \\
1 & Sentence Completion \\
2 & Different Word \\
3 & Word Analogies \\
4 & Superordinates \\
5 & Categorical Memory \\
6 & Arithmetic Problems \\
7 & Numerical Progression \\
8 & Figures \\
9 & Cube Matching \\
Achievement & Tests \\
10 & German \\
11 & History \\
12 & Geography \\
13 & English \\
14 & French \\
15 & Mathematics \\
16 & Physics \\
17 & Chemistry \\
18 & Biology \\
19 & Arts \\
20 & Music \\
\hline
\end{tabular}

a positive constant to all the given distances, to satisfy the triangle inequality, and therefore it should be evaluated using measures that are unaffected by such a transformation. These values, for all test representations, are shown in the top part of Table 5 , while the corresponding values for the representations of items (of Dataset A) are shown in the lower part of Table 5. Recall that in ADDTREE the distances between the objects are determined by $2 n-2$ parameters at most; SSA/2D has $2 n$ parameters at most; SSA/3D has $3 n$ parameters (where $n$ is the number of elements in the set).

\section{Results}

\section{Goodness of Fit}

The results displayed in Table 5 indicate that ADDTREE has an advantage over SSA/2D in two out of the three datasets, and in one case, it even fits the data better than SSA/3D although the latter is based on a larger number of parameters. The results regarding representation of items are even clearer. In all 14 groups, both the linear fit and the monotonic fit of ADDTREE are higher than that of SSA $2 \mathrm{D}$, and in all but one group the linear fit of ADDTREE is higher even than the fit of SSA/3D. When a monotone measure was considered, the advantage of ADDTREE over SSA/3D was found in nine out of the 14 groups. ${ }^{1}$

\section{Interpretation}

The application of each model to a given dataset results in a configuration representing the distances between the variables (whether tests or items). Due to space limitations, only examples from the first two datasets are considered.

\footnotetext{
'SSA does not assume a linear relationship between the data and the solution and therefore $R_{M}^{2}$ is a more appropriate goodnessof-fit measure for that model. The SSA solution minimizes its own stress formula, the coefficient of alienation (COA; see Lingoes, 1970). This measure is similar in principle to the $R_{M}^{2}$ measure and was also computed for SSA and ADDTREE. The ADDTREE solution yielded smaller COA values (indicating better fit) in all datasets.
} 
Table 5

Goodness-of-Fit Results for ADDTREE, the

Two-Dimensional SSA Solution (SSA/2D), and the

Three-Dimensional SSA Solution (SSA/3D)

\begin{tabular}{|c|c|c|c|c|c|c|}
\hline \multirow[b]{2}{*}{ Dataset } & \multicolumn{2}{|c|}{ ADDTREE } & \multicolumn{2}{|c|}{$\mathrm{SSA} / 2 \mathrm{D}$} & \multicolumn{2}{|c|}{$\mathrm{SSA} / 3 \mathrm{D}$} \\
\hline & $R_{L}^{2}$ & $R_{M}^{2}$ & $R_{L}^{2}$ & $R_{M}^{2}$ & $R_{L}^{2}$ & $R_{M}^{2}$ \\
\hline PET & .94 & .95 & .84 & .92 & .94 & .98 \\
\hline Validation Study & .90 & .94 & .90 & .95 & .95 & .99 \\
\hline Hoger's Study & .81 & .88 & .74 & .87 & .77 & .89 \\
\hline \multicolumn{7}{|c|}{ PET Relatively Heterogeneous Items } \\
\hline a & .83 & .85 & .71 & .79 & .77 & .84 \\
\hline$b$ & .84 & .86 & .71 & .78 & .79 & .84 \\
\hline$c$ & .89 & .90 & .77 & .86 & .84 & .91 \\
\hline$d$ & .92 & .94 & .64 & .80 & .75 & .87 \\
\hline e & .85 & .87 & .79 & .84 & .86 & .90 \\
\hline 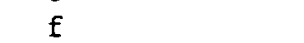 & .84 & .86 & .62 & .73 & .73 & .82 \\
\hline \multicolumn{7}{|c|}{ Relatively Homogene } \\
\hline 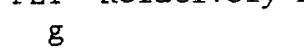 & .72 & .76 & .53 & .58 & .67 & .71 \\
\hline h & .86 & .89 & .75 & .84 & .80 & .87 \\
\hline$i$ & .87 & .90 & .78 & .87 & .86 & .90 \\
\hline j & .85 & .88 & .77 & .86 & .84 & .91 \\
\hline $\mathrm{k}$ & .97 & .98 & .92 & .98 & .96 & .99 \\
\hline 1 & .88 & .90 & .75 & .82 & .80 & .85 \\
\hline $\mathrm{m}$ & .89 & .92 & .63 & .86 & .69 & .89 \\
\hline $\mathrm{n}$ & .93 & .95 & .60 & .96 & .81 & .96 \\
\hline
\end{tabular}

The grouping of the objects into clusters is derived directly from the ADDTREE solution. On the other hand, the SSA solution provides a configuration of points in a space, but the grouping of the objects into clusters must be carried out separately.

Note that the interpretation of dimensions in MDS and clusters in ADDTREE is not part of the formal analysis. It is subjective and open to further discussion and debate. Construction of formal representations always incurs the question of how much of the formal solution should be interpreted (e.g., the labeling of clusters in cluster analysis, factors in factor analysis, and dimensions in MDS). The labeling of the arcs in ADDTREE represents an attempt to facilitate the understanding of the configuration by providing interpretation in terms of common and distinctive features. Following the tradition in the scaling literature (e.g., Shepard, 1980), only the major and the most distinct clusters were labeled. In ADDTREE, labels were given to arcs relatively high on the hierarchy and to those which are relatively long.
Figure 1 presents the ADDTREE solution for the 21 subtests included in Dataset $\mathrm{A}$. The subtests are grouped into hierarchically ordered clusters. It can be seen that the tests are partitioned into two major clusters that can be labeled "problem solving tests" (i.e., tests that require inductive and deductive thinking) and "tests of knowledge" (i.e., tests of verbal ability, such as Vocabulary or English, and tests of General Knowledge). The length of the arc labeled "problem solving" can be viewed as representing the common features shared only by the tests clustered under this arc (Tests 8-16), while the arc labeled "knowledge" represents the measure of the common features shared by the tests that cluster under it, and by them alone.

Each of these major clusters is further partitioned into subclusters. For example, the arc labeled "Math" can be viewed as the measure of common features shared only by the subcluster of the three tests that emerge from it (Tests 9-11). These are mathematical reasoning tests that rely on high-school mathematical subject matters. This subcluster fur- 
Figure 1

ADDTREE Representation of the PET Tests (Dataset A)

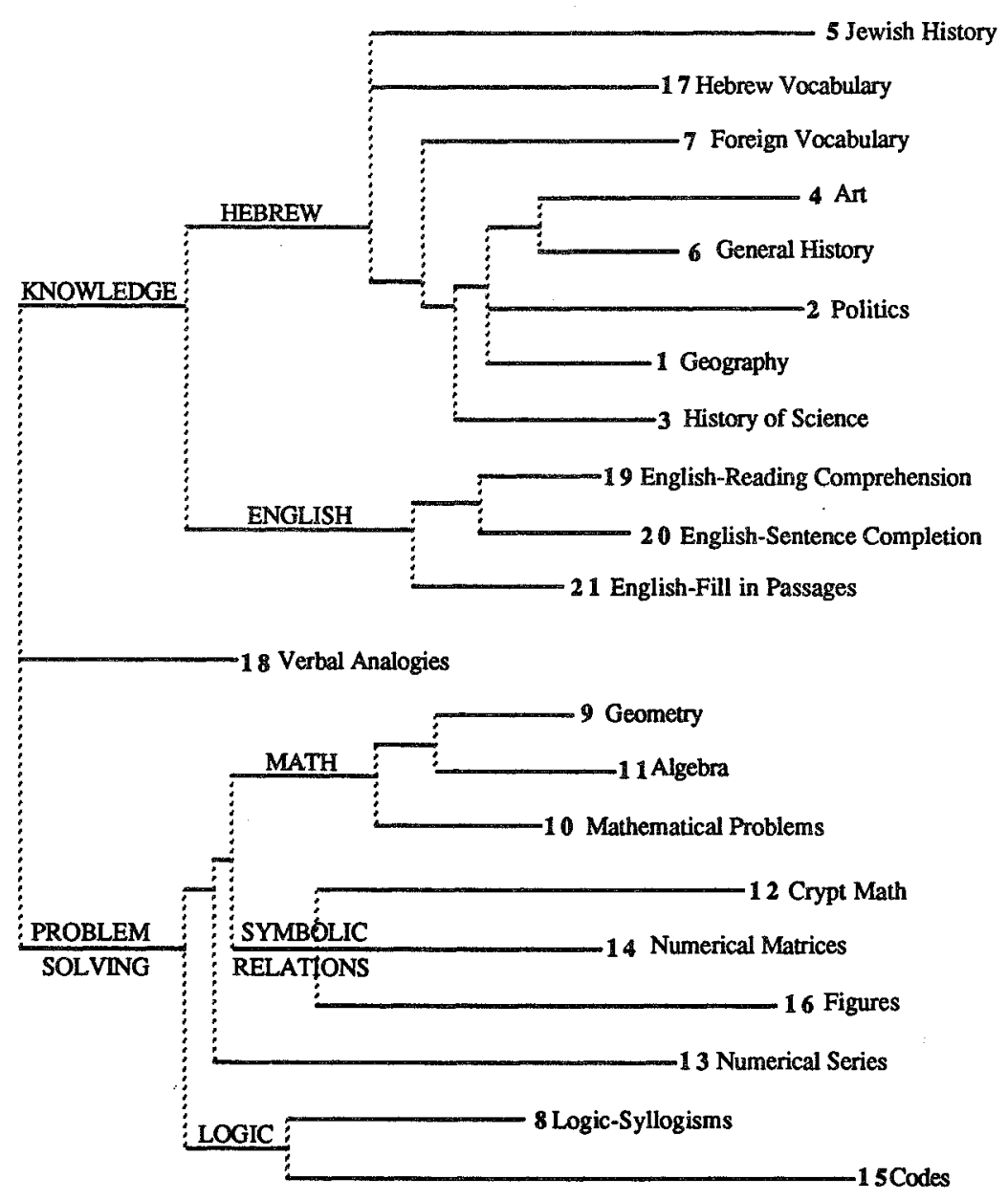

ther divides into two additional subclusters: Math Problems tests (which require translation of a verbal problem into mathematical terms) and Geometry and Algebra tests. Clearly ADDTREE provides further subdivisions into smaller and more specific clusters. A full interpretation of the tree requires a thorough analysis of each subtest or even of individual items. Such an analysis is beyond the scope of this article.

The terminal arcs of the tree correspond to the measure of the unique features of the respective tests. For example, the terminal arc of Test 11 represents the unique features of Algebra items not shared by any other subtest, not even by its close neighbor-Geometry.

The Verbal Analogies test is connected to the root of the tree by a relatively short arc, and is therefore "central" in the sense that it is close to both major clusters. Recall that centrality in a tree is represented by a short arc, which reflects few distinctive features (Tversky \& Hutchinson, 1986). The distance between any two tests in a tree is the (horizontal) length of the path that connects them. Therefore, a test that is closest to the root of the 
tree has the minimal average distances from all other tests. Indeed, this representation of the Verbal Analogies test is consistent with the notion that this test measures general ability and requires both "reasoning" and "knowledge." Note that the commonality shared by the Verbal Analogies test and all other tests is not represented by a specific arc in the tree. Rather than general commonality, the tree represents the commonality among objects of a certain subcluster relative to the rest.

The two major clusters in the tree correspond to the "operation facet"' suggested by Guttman (1965). The evidence, however, seems incompatible with the other facet, "language of presentation", suggested by Guttman. For example, the Figure test and the Numerical Matrices test are clustered together despite their different language of presentation. At the same time, the Logic test, which is verbal, does not cluster together with the other verbal ability tests although they share "language of presentation." It appears that the cognitive operation explains more of the variance in the correlations among the tests than does the mode of presentation.

The SSA/2D solution (Figure 2) produces the same two major clusters. They are reconstructed mainly along one dimension that can be labeled "cognitive operation." The Verbal Analogies test is located in the middle. The subdivision within each cluster is hardly seen, especially within the reasoning cluster.

As can be expected by its greater number of parameters, the SSA/3D solution improves the fit (as compared with the SSA/2D)-but it is more difficult to grasp, and apparently does not add much to the interpretation.

For the purpose of demonstration, one group of items was selected to represent the analyses of the item correlation matrices (Dataset $\mathrm{C}$ in Table 2). This particular group consists of 42 items that are relatively heterogeneous in content and of an intermediate level of difficulty. The configuration extracted from the analyses of these items is very similar to those derived from the analyses of the subtests (Figures 1 and 2). The items, like the subtests, cluster into two major groups: "problem solving"' and "knowledge".

The ADDTREE solution (Figure 3) represents a clear hierarchical structure of the items, in which each cluster is subdivided into subclusters. The resemblance between the items' structure and the tests' structure is revealed not only in the above two major clusters but also in the further division into subclusters. It is interesting to note that all

Figure 2

SSA-I Representation of the PET Tests (Dataset A)

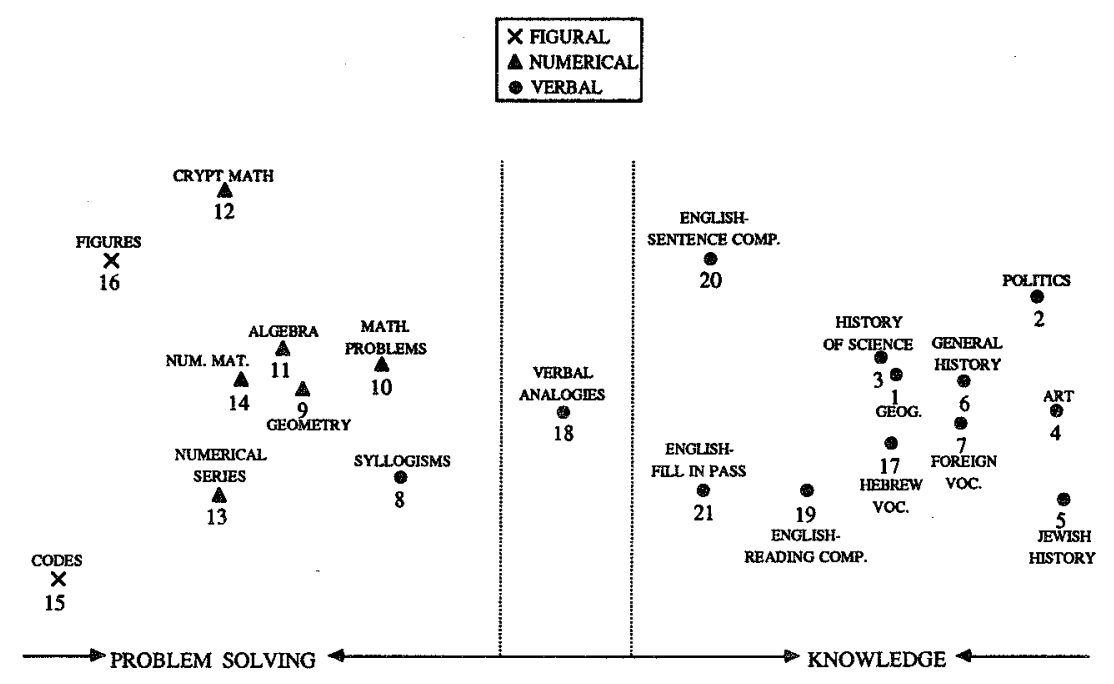


Figure 3

ADDTREE Representation of One Set of Items (Set C) Taken From the PET

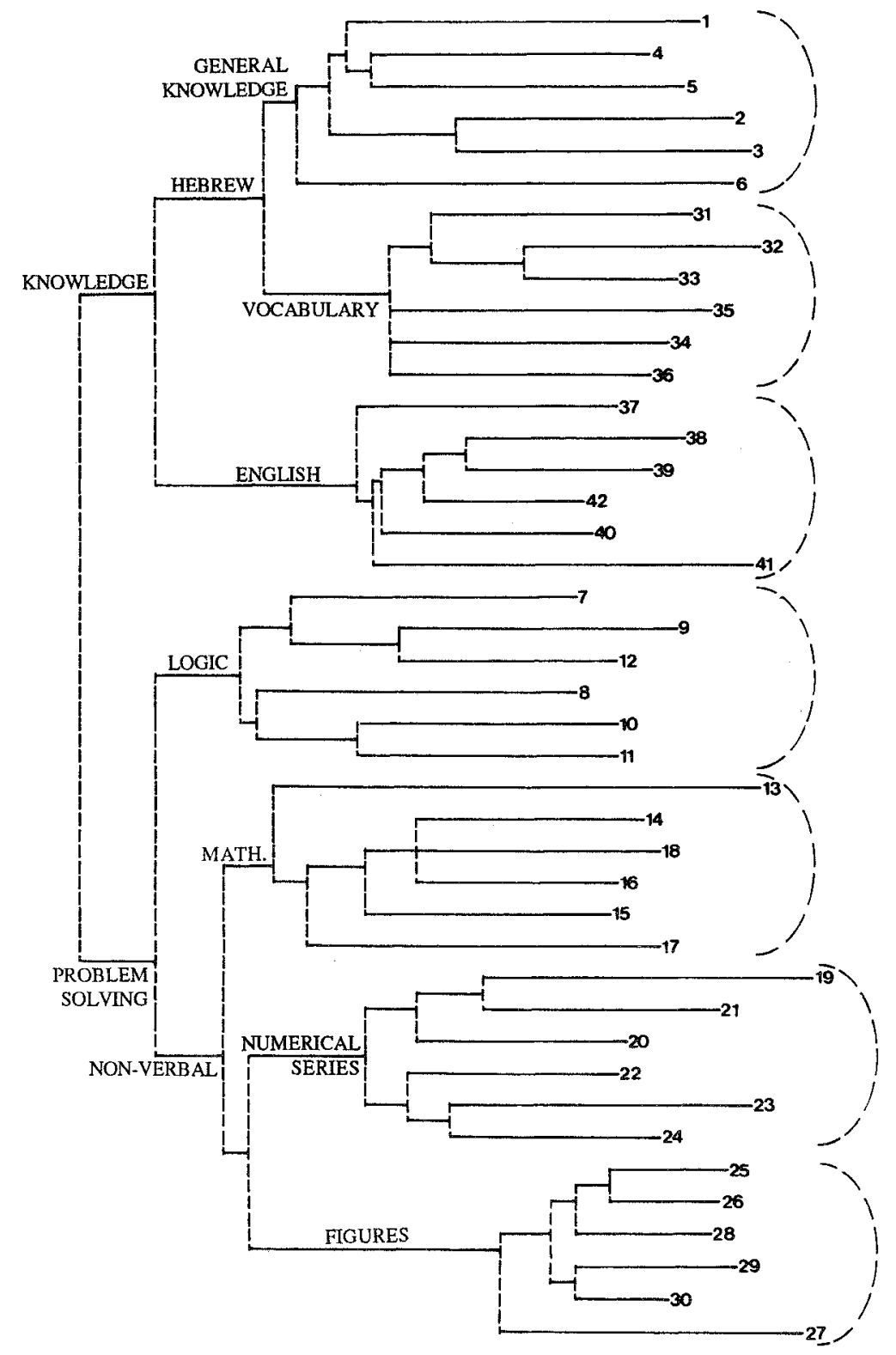

items of a certain subtest are always clustered together. Such perfect clustering of the individual items into their corresponding subtests is nontrivial, especially because individual items have relatively low reliabilities. This result strengthens the content and construct validity of the psychometric test.

The SSA/2D solution, presented in Figure 4, clearly reflects the distinction between the same two clusters: "knowledge" items and "problem solving" 
Figure 4

SSA-I Representation of One Set of Items (Set C) Taken From the PET

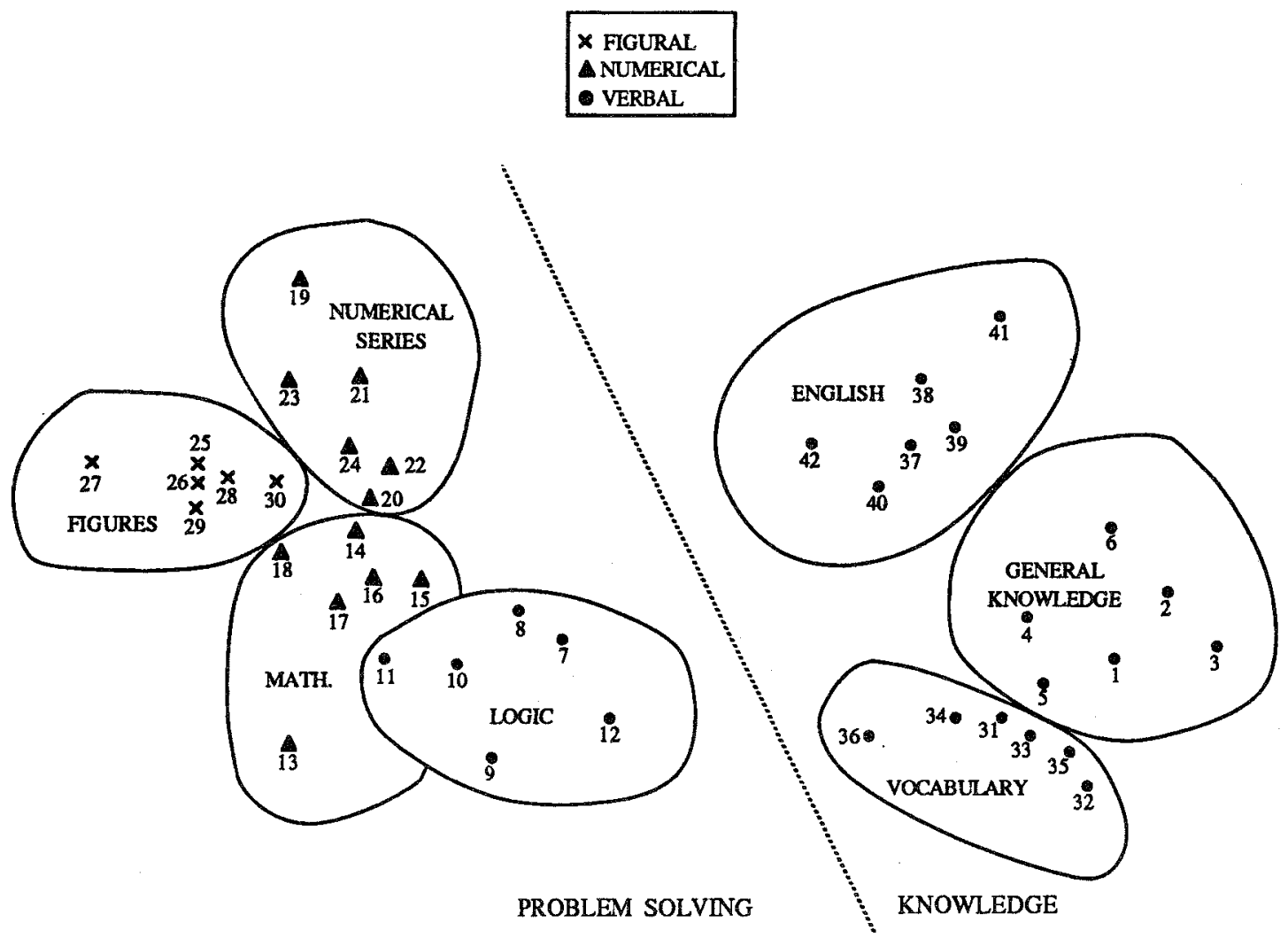

items. Within each cluster the items can be grouped according to the original subtests from which they were extracted. This grouping, however, is not given directly by the algorithm, and it is somewhat less distinct than in ADDTREE (e.g., Math and Logic clusters). The horizontal axis can be interpreted as representing the difficulty level of the items.

Figure 5 presents the ADDTREE solution for the 12 subtests included in Dataset B. Recall that this study consisted of both general aptitude and achievement tests. This typology is clearly reflected in all of the pictorial representations. In the ADDTREE solution (Figure 5), the major clusters are further divided into subclusters. The subclusters within the aptitude cluster give rise to a structure very similar to the PET structure described earlier. This finding suggests that the representation ob- tained by ADDTREE for the aptitude tests is invariant to the addition of variables of a different nature (e.g., achievement tests). It is not clear whether this finding will generalize to other types of variables.

The second major cluster-achievement-is further divided into the criterion and the matriculation tests. The criterion is represented by a relatively short branch, and therefore can be seen as the central or typical variable. This is compatible with the fact that all other variables serve as predictors of the criterion in this validation study. Note that the length of the tree path between each predictor and the criterion variable reflects its respective predictive validity.

Figure 6 presents the SSA/2D solution for these 12 subtests. This is the only dataset in which SSA 
Figure 5

ADDTREE Representation of the Variables in the Validation Study (Dataset B)

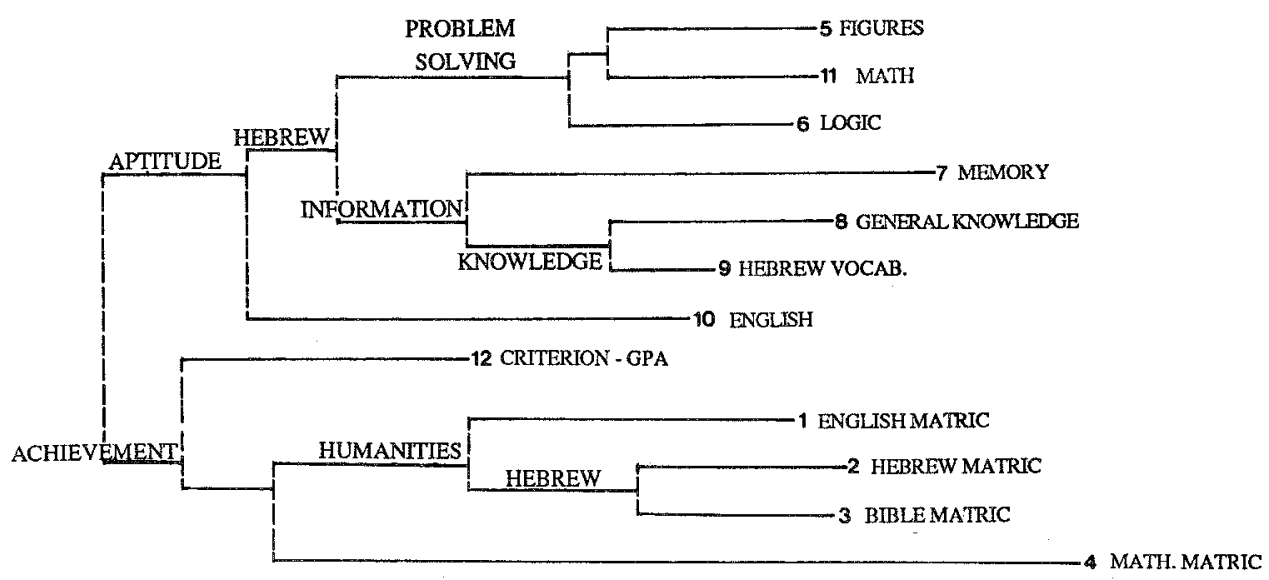

yielded a goodness-of-fit measure equivalent to that of ADDTREE. It seems that these data are the product of two orthogonal factors: (1) problem solving vs. knowledge; (2) aptitude vs. achievement. The criterion lies in the middle of the two-dimensional space.

Dataset $\mathrm{C}$ will be discussed without the benefit of the accompanying figures. As in Dataset $B$, this study included both general aptitude and achieve- ment tests, and this feature was again clearly reflected by the major clustering of the pictorial solutions. In ADDTREE, the general aptitude cluster was subdivided into verbal tests (Tests 1-3), categorization tests (Tests 4 and 5), and nonverbal tests (Tests 6-9). In this dataset, the language of presentation and the type of cognitive operation were confounded (e.g., all verbal tests are reasoning tests). Therefore, the interpretation is some-

Figure 6

SSA-I Representation of the Variables in the Validation Study (Dataset B)

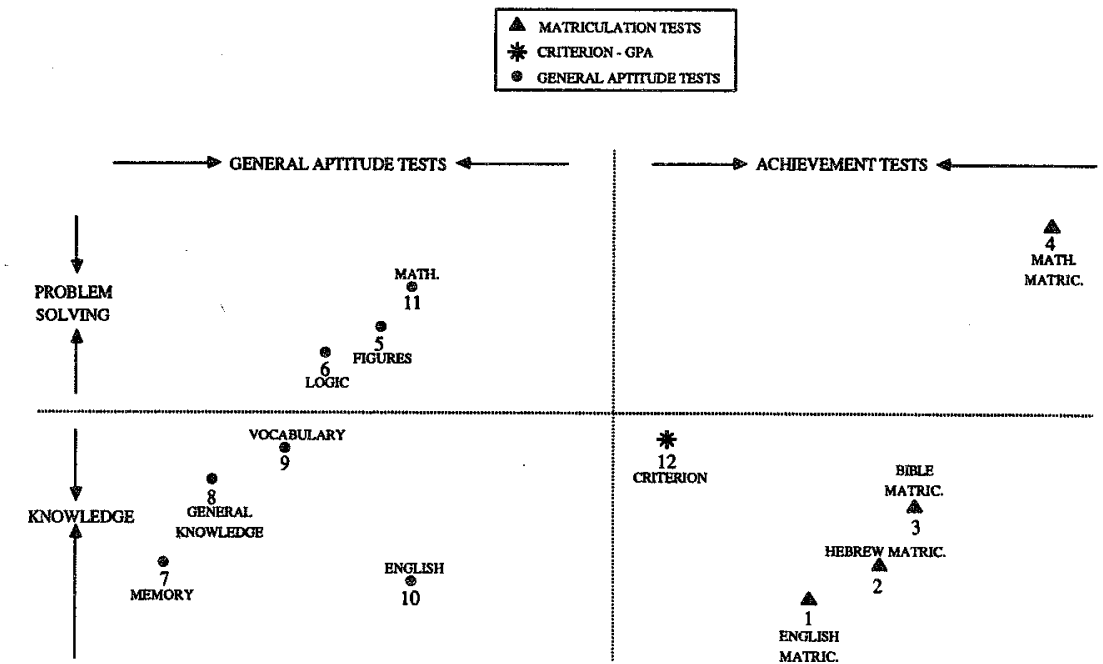


what ambiguous and the verbal/nonverbal distinction could be replaced by a reasoning/perception distinction. The achievement cluster was subdivided into languages (Tests 10,13 , and 14), arts (Tests 19 and 20), and other high school studies (Tests 11, 12, 15, 16, 17, and 18).

In the SSA/2D solution, the radex structure extracted by Schlesinger and Guttman (1969) for 18 variables (out of the 20 variables in the original study) was not replicated when all 20 variables were used in the analysis. The inclusion of the two additional variables (art and music) changed the structure within the remaining 18 variables. This casts some doubt on the radex theory as a general structure of the interrelations between tests, and especially as a general theory of intelligence.

\section{Comparison With Principal Components Analysis}

Due to space limitations, only the results for Dataset A are presented. The measure $R_{L}^{2}$, used earlier to indicate goodness of fit, was computed for the principal components analyses with two and three components (2PC and 3PC), yielding values of .86 and .95 respectively. These results are very similar to the $R_{L}^{2}$ measures obtained for SSA with two and three dimensions, respectively (see Table 5). ADDTREE's goodness-of-fit measures were similar to the 3PC solutions in this dataset as well as in all other datasets.

Unlike MDS and ADDTREE, factor-analytic techniques are designed to reproduce the zero point of the correlations. The goodness-of-fit measures used for the present analyses $\left(R_{M}^{2}\right.$ and $\left.R_{L}^{2}\right)$ are not sensitive to the zero point and therefore do not capture this feature in the data. A goodness-of-fit measure that is sensitive to the zero point (e.g., coefficient of congruence; Harman, 1976, p. 344) would obviously be more favorable to the principal components analysis, but would be meaningless to SSA and ADDTREE as explained earlier. Clearly, when it is desired to reproduce the absolute value of the correlations, factor-analytic techniques will be preferred.

The loadings of the 21 subtests of Dataset A on the three components (after varimax rotation) are presented in Table 6 . The first component represents General Knowledge tests and Hebrew Vocabulary. The second component represents Problem Solving tests (verbal, numerical, and figural reasoning tests). The Verbal Analogy test is loaded on both factors, which demonstrates again the "centrality" of this variable. The third component is a specific component representing the three English subtests (testing English as a foreign language). It seems that the three components correspond to the major clusters revealed by both ADDTREE and SSA/2D. However, only ADDTREE representation reveals the further division of each cluster into subgroups.

\section{Discussion}

The present study demonstrates the utility of applying a clustering analysis, ADDTREE, to represent the structures of tests and test items on the basis of their intercorrelations. In general, the same major clusters emerged for all datasets analyzed and under all representation models used. This major clustering corresponds to the cognitive operation required for solving the test items (i.e., "reasoning" vs. "knowledge"). The language of presentation, which is a major factor in Guttman's (1965) and Guilford's (1967) theories, does not seem to add much to the interpretation. This may be partly because in many cases the language is confounded with the mode of cognitive operation (e.g., tests that require knowledge or rule application are usually verbal).

ADDTREE fared well in the present comparisons. First, it fit the data better than SSA-I (with two dimensions). Moreover, the grouping of the objects (in this case tests and items) into clusters and subclusters in ADDTREE is straightforward and less dependent on the specific interpretation given by the researcher. By comparison, the SSA solution does not directly provide clusters; they must be extracted by the researcher without the benefit of an algorithm. SSA, as a method of MDS, is beneficial to the extent to which its dimensions are interpretable. In most datasets analyzed here, it was difficult to 


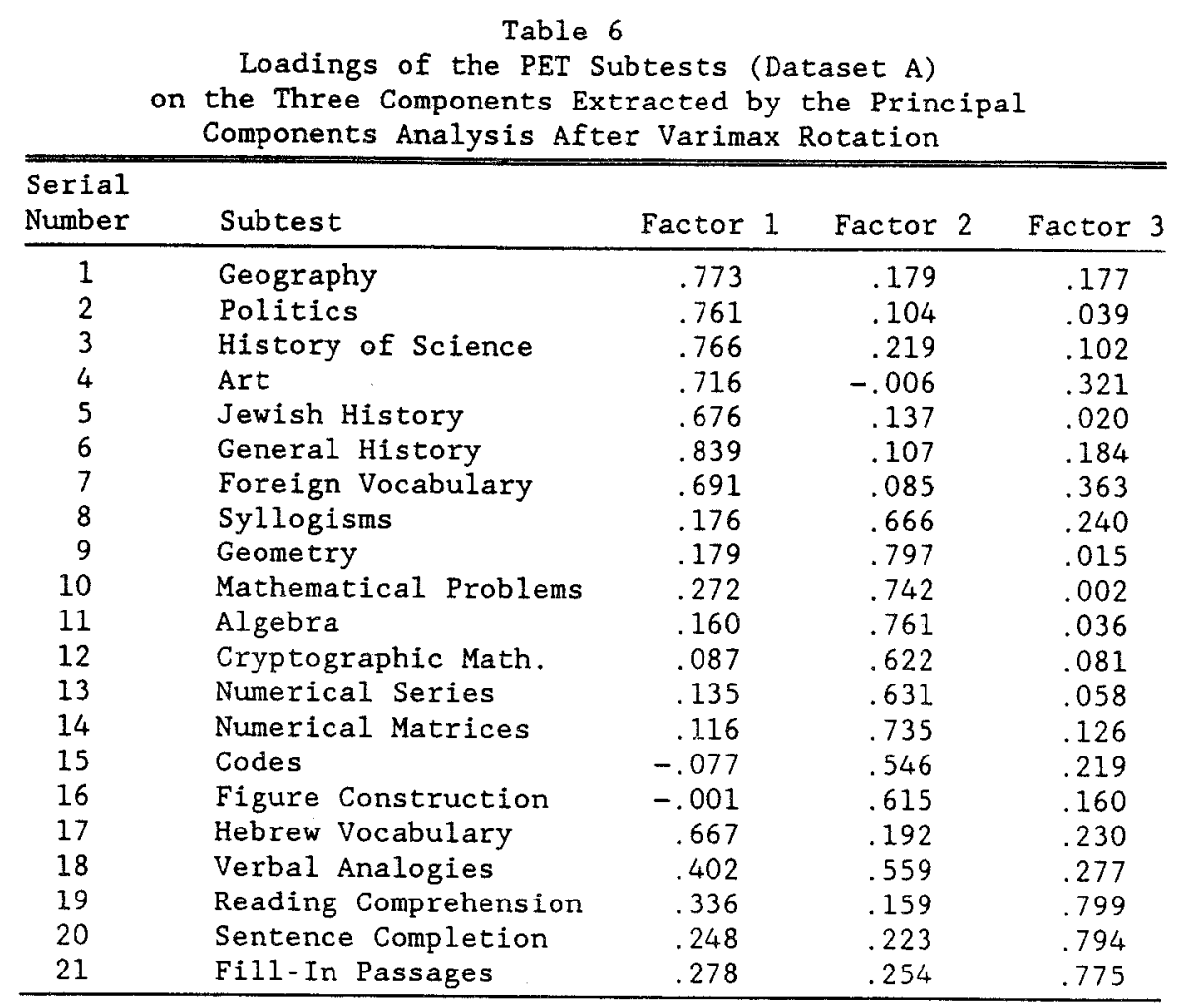

find meaningful interpretations for the linear dimensions. To the extent that such an interpretation is given, it does not use the dimension's continuity, but rather leans toward a division of the space into discrete regions. The ADDTREE solution, on the other hand, provides a formal procedure for identifying clusters and lends itself to an interpretation in terms of hierarchical structure. The present data analyses show that the radex structure suggested by Guttman (1965) is not stable across different datasets and within datasets when variables are deleted or added.

The principal components analyses (with three components) explained the variance among the subtests well and yielded high goodness-of-fit measures (similar to those obtained by ADDTREE). This justifies the extensive use of this method in psychological testing. The interpretation derived from the principal components analyses is compatible with that derived from ADDTREE, although it does not reveal the hierarchical structure that emerged from the trees. It seems that apart from clustering, no significant interpretation could be assigned to the components of the principal components analyses or to the linear dimensions of SSA. The only salient result is that the division of the tests according to their loadings on the different factors closely paralleled the clustering revealed by ADDTREE. Further research may be required to enable a more thorough comparison of tree structure to factor-analytic results.

In general, ADDTREE provides a parsimonious method for representing datasets characterized by a relatively large number of orthogonal dimensions (such that each dimension has a relatively large unique variance). The fit of ADDTREE to the present datasets may suggest that the objects represented (tests and items) have those characteristics that have been found by Tversky and Hutchinson (1986) to favor a tree over a spatial representation (i.e., the objects are characterized by a large number of discrete or specific features, rather than a small number of continuous dimensions).

Despite the long tradition of using spatial rep- 
resentations as the only formal means of representing the relations between tests and items, tree models in general, and ADDTREE in particular, may serve as an efficient and promising approach. Its close fit to the data and its coherence of presentation make ADDTREE a convenient means of representing individual differences in cognitive tasks. One possible interpretation of this representation could be based on the "contrast model" (Tversky, 1977). Application of the contrast model to the relationship among tests and items requires an assumption that test items can be characterized by a set of discrete features (common and distinct), such that the similarity between the variables (reflected by the correlation between them) increases with their common features. Ongoing experimental research designed to discover cognitive processes involved in problem solving (e.g., Sternberg, 1977, 1980) can make use of this type of representation.

Another implication of the present study can be drawn from a psychometric point of view. ADDTREE analysis of the items yielded a clustering structure of the individual items that fully reconstructed the original subtests from which the items were extracted as well as the interrelations among those subtests. This provides corroboration of the construct and content validity of the test, and may suggest that the application of ADDTREE analysis to the representation of items can be used in order to assist the process of test construction and item analysis. For example, an item that does not cluster with the other items designed to measure the same construct should be further examined and its inclusion in a test should be reconsidered. An item that is very distant from the root should be checked, because it either lacks reliability or measures unique features not shared by other items.

In the present example (see Figure 3) all items of a given subtest cluster together, but in some cases clusters subdivide such that one item seems to deviate from other items in its cluster. For instance, Item 13 seems to deviate from the rest of the mathematical items, although it falls within the Math cluster. This is important and relevant information for test constructors, who ought to look more closely into these items and examine possible reasons for such occurrences.
Hierarchical representation of tests is highly suggestive and may offer a starting point for theories about the structure of the intellect, or at least the structure of mental abilities. The hierarchical structure suggested here by ADDTREE fits some current theories of the intellect (e.g., Cattell, 1971; Snow, 1978). On the other hand, the transition from the analyses of correlations across persons to a theory of intelligence is a major challenge that has not been satisfactorily answered nor fully articulated. The bridge between them must be built rather than prejudged.

\section{References}

Beller, M., \& Ben-Shakhar, G. (1981). An evaluation study of the students' selection process at the Hebrew University of Jerusalem. Megamot-Behavioral Sciences Quarterly, 27, 22-36.

Carroll, J. D. (1976). Spatial, non-spatial and hybrid models for scaling. Psychometrika, 41, 439-463.

Carroll, J. D., \& Pruzansky, S. (1980). Discrete and hybrid scaling models. In E. D. Lantermann \& $\mathbf{H}$. Feger (Eds.), Similarity and choice. Bern: Huber.

Cattell, R. B. (1971). Abilities: Their structure, growth and action. Boston: Houghton-Mifflin.

Coombs, C. H. (1964). A theory of data. New York: Wiley.

Corter, J. E. (1982). ADDTREE/P: A PASCAL program for fitting additive trees based on Sattath and Tversky's ADDTREE algorithm. Behavior Research Methods and Instrumentation, 14, 353-354.

Davison, M. L. (1983). Multidimensional scaling. New York: Wiley.

Davison, M. L. (1985). Multidimensional scaling versus components analysis of test intercorrelations. Psychological Bulletin, 97, 94-105.

Gati, I. (1979). A hierarchical model for the structure of vocational interests. Journal of Vocational Behavior, $15,90-106$.

Gati, I. (1982). Testing models for the structure of vocational interests. Journal of Vocational Behavior, 21, 164-182.

Guilford, J. P. (1967). The nature of human intelligence. New York: McGraw-Hill.

Guttman, L. (1965). A faceted definition of intelligence. In R. Eiferman (Ed.), Studies in psychology, Scripta Hierosolymitana 14 (pp. 166-181). Jerusalem: Hebrew University.

Guttman, L. (1968). A general nonmetric technique for finding the smallest coordinate space for a configuration of points. Psychometrika, 33, 469-506.

Harman, H. H. (1976). Modern factor analysis (3rd ed.). Chicago: University of Chicago Press. 
Hayes, W. L. (1981). Statistics (3rd ed.). New York: Holt, Rinehart and Winston.

Hoger, D. J. (1964). Analyse der Intelligenzstruktur bei mannlichen-Gymnasiasten der Klassen 6-9 (Unter Sekunda-Oberprima). Psychologische Forschung, 27, 419-474.

Horn, J. L. (1968). Organization of abilities and the development of intelligence. Psychological Review, 75, 242-259.

Johnson, E. J., \& Tversky, A. (1984). Representations of perceptions of risks. Journal of Experimental Psychology: General, 113, 55-70.

Johnson, S. C. (1967). Hierarchical clustering schemes. Psychometrika, 32, 241-254.

Kruskal, J. B. (1964). Multidimensional scaling by optimizing goodness of fit to a non-metric hypothesis. Psychometrika, 29, 1-27.

Kruskal, J. B., Young, F. W., \& Seery, J. B. (1977). How to use KYST.2A, a very flexible program to do multidimensional scaling and unfolding. Unpublished manuscript, AT\&T Bell Laboratories.

Lingoes, J. C. (1970). An IBM 360/67 program for Guttman-Lingoes smallest space analysis-PI. Behavioral Science, 15, 536-540.

Pruzansky, S., Tversky, A., \& Carroll, J. D. (1982). Spatial versus tree representations of proximity data. Psychometrika, 47, 3-24.

Sattath, S., \& Tversky, A. (1977). Additive similarity trees. Psychometrika, 42, 319-345.

Sattath, S., \& Tversky, A. (1987). On the relation between common distinctive feature models. Psychological Review, 94, 16-22.

Schlesinger, I. M., \& Guttman, L. (1969). Smallest space analysis of intelligence and achievement tests. Psychological Bulletin, 71, 95-100.

Shepard, R. N. (1962a). The analysis of proximities: Multidimensional scaling with an unknown distance function. Part I. Psychometrika, 27, 125-140.

Shepard, R. N. (1962b). The analysis of proximities: Multidimensional scaling with an unknown distance function. Part II. Psychometrika, 27, 219-246.

Shepard, R. N. (1972). Introduction to Volume I. In R. N. Shepard, A. K. Romney, \& S. B. Nerlove (Eds.), Multidimensional scaling theory and applications in the behavioral sciences (Vol. I). New York: Seminar Press.

Shepard, R. N. (1974). Representation of structure in similarity data: Problems and prospects. Psychomet- rika, 39, 373-421.

Shepard, R. N. (1980). Multidimensional scaling, treefitting and clustering. Science, 210, 390-398.

Shepard, R. N., \& Arabie, P. (1979). Additive clustering: Representation of similarities as combinations of discrete overlapping properties. Psychological $R e$ view, 86, 87-123.

Snow, R. E. (1978). Theory and research on aptitude processes. Intelligence, 2, 225-278.

Spearman, C. (1927). The abilities of man. New York: Macmillan.

Sternberg, R. J. (1977). Intelligence, information-processing, and analogical reasoning: The componential analysis of human abilities. Hillsdale NJ: Erlbaum.

Sternberg, R. J. (1980). Sketch of a componential subtheory of human intelligence. Behavioral and Brain Sciences, 3, 573-584.

Thurstone, L. L. (1938). Primary mental abilities. Psychometric Monograph, No. 1.

Tryon, T. C., \& Bailey, D. E. (1970). Cluster analysis. New York: McGraw-Hill.

Tversky, A. (1977). Features of similarity. Psychological Review, 84, 327-352.

Tversky, A., \& Hutchinson, J. W. (1986). Nearest neighbor analysis of psychological spaces. Psychological Review, 93, 3-22.

Tversky, A., \& Sattath, S. (1979). Preference trees. Psychological Review, 86, 542-573.

Vernon, P. E. (1961). The structure of human abilities (2nd ed.). London: Methuen.

\section{Acknowledgments}

The author thanks Herve Abdi, Maya Bar-Hillel, David Budescu, Itamar Gati, Amos Tversky, and two anonymous reviewers for their helpful comments and suggestions, as well as the Division of Life Sciences at the University of Toronto, Scarborough Campus, for providing the facilities for writing this article during the author's sabbatical.

\section{Author's Address}

Send requests for reprints or further information to Michal Beller, National Institute for Testing and Evaluation, 4 Rav Ashi Street, Jerusalem, Israel. 